\title{
Effect of Moisture Content and Inoculum on the Growth and Conidia Production by Beauveria bassiana on Wheat Bran
}

\author{
Oscar Nuñez-Gaona ${ }^{1}$, Gerardo Saucedo-Castañeda ${ }^{1}$, Raquel Alatorre-Rosas ${ }^{2}$ and Octavio \\ Loera $^{1 *}$ \\ ${ }^{1}$ Universidad Autónoma Metropolitana-Iztapalapa; Departamento de Biotecnologia; 09340; Mexico D.F. - Mexico. \\ ${ }^{2}$ Colegio de Posgraduados; Instituto de Fitosanidad; Montecillo, 56230; Edo. de México - Mexico
}

\begin{abstract}
The aim of the present work was to study the effect of moisture content and inoculum on the growth and conidia production by Beauveria bassiana on wheat bran (WB).The highest growth rate of $\mathrm{B}$. bassiana 885.2 on WB media was obtained at $a_{w}=1.0$, with no detected growth at $a_{w}<0.97$. Solid-state fermentation (SSF) using WB (66\% moisture; $\left.a_{w}=1.0\right)$ achieved a maximal yield of $1.18 \times 10^{10}$ conidia per gram of dry substrate (gds). This yield decreased one order of magnitude with higher moisture contents or the addition of sugarcane bagasse (SCB) as a texturiser. In SSF using WB (66\% humidity), the time to obtain a yield of $1 \times 10^{10}$ conidia/gds, referred to as $t_{10}$, could be predicted using a model considering common inoculum levels and maximal yields. For instance, $t_{10}$ was $285 \mathrm{~h}$ with an inoculum of $1 \times 10^{6}$ conidia/gds; however, $t_{10}$ was reduced to $232 \mathrm{~h}$ and 148 for inocula of $7 \times 10^{6}$ and $5 \times 10^{7}$ conidia/gds, respectively. The estimation of $t_{10}$ values allowed both comparison between the cultures and prediction of harvesting times in production processes. Values for hydrophobicity were within 90 and $92 \%$, whereas viability averages were around $70 \%$ for all the cultures
\end{abstract}

Key words: Beauveria bassiana; wheat bran; water activity; texturiser; conidial yields

\section{INTRODUCTION}

Organic farming requires practical and suitable approaches to substitute the chemical pesticides used in crop fields worldwide. Biological control is considered an important part of integral pest management (IPM), which also is an environmental friendly alternative (Fuxa 1987). Beauveria bassiana is widely extended in nature; this fungus has the potential to control a wide range of insect pests. Additionally, it is innocuous for human and other mammals (Fuxa 1987; Arcas et al. 1999). Interest for industrial production has increased over the past few years, and in fact, there are commercial products based on conidiospores of this fungus (Fuguest and Vey 2004).

In order to produce conidia of entomopathogenic fungi at industrial level, there are two main methods, submerged fermentation $(\mathrm{SmF})$ and solid-state fermentation (SSF); however in SmF, entomophatogenic fungi mainly produce blastospores and conidia yields are low (Feng et al. 2000). Conversely, in SSF, the growth of microorganisms is achieved over solid supports

*Author for correspondence: loera@xanum.uam.mx 
with substantially low levels of water (Pandey 2003). There are some key factors affecting the growth and conidial production in SSF and it is important to know their influence in the processes. Some of these main factors are temperature, $\mathrm{pH}$, substrate (type and concentration), and moisture content (Barranco-Florido et al. 2002; Larroche 1996; Arzumanov et al. 2005).

The aim of this work was to evaluate the effect of moisture content and inoculum size on the growth and conidia production by Beauveria bassiana using wheat bran as a substrate. Sugar cane bagasse as a texturiser was also analyzed in these cultures. Also, a criterion was proposed based on times for reaching suitable conidial yields, which could be relevant for the prediction of harvesting times and for proper culture comparison.

\section{MATERIALS AND METHODS}

\section{Microorganism propagation}

The strain of Beauveria bassiana 885.2 was propagated on 4\% (w/v) Dextrose Sabouraud Agar (DSA). This strain belongs to the Universidad Autonoma Metropolitana culture collection. Conidia were harvested after cultivation for 7 to 10 days at $28 \pm 0.5^{\circ} \mathrm{C}$ and stirring the culture with a $0.05 \%(\mathrm{v} / \mathrm{v})$ aqueous solution of Tween 80 for 10 min. For both conidial density and radial extension rate assays, conidia were point inoculated in triplicate in the center of Petri dishes containing wheat bran (10\%) as the only nutrient source plus agar $(15 \mathrm{~g} / \mathrm{L})$. This medium was also supplemented either with components of a synthetic medium (Alves et al. 2002) or standard 4\% (w/v) Dextrose Sabouraud broth diluted (1:2) previously. Ethylene glycol (0 to 20\%) was used as a water activity $\left(\mathrm{a}_{\mathrm{w}}\right)$ depressor in these media (Montiel-González et al. 2004), which allowed the modification of $a_{w}$ values within a range of 1.0 to 0.93. Water activity was evaluated using a hygrometer (Acualab, Cx-2, Labsenscientific Co, USA, 1952). All media were sterilized at $15 \mathrm{psi}$ $\left(121.5^{\circ} \mathrm{C}\right)$ for $15 \mathrm{~min}$. Inoculated cultures were incubated at $28 \pm 0.5^{\circ} \mathrm{C}$ for 14 days, then conidia were harvested and counted.

\section{Solid-state fermentation}

One gram of wheat bran was placed in $75 \mathrm{~mL}$ serological bottles. Moisture was adjusted to 66 , 75 and $80 \%$ by adding 2,3 and $4 \mathrm{~mL}$ of distilled water, respectively. Half of the proper amount of water for routine experiments was added prior to sterilization; the rest of water was added afterwards, containing conidial inoculum and Dextrose Sabouraud broth as described earlier. Three bottles for each moisture value were inoculated with a final concentration of $5 \times 10^{7}$ conidia per gram of initial dry substrate (gds). Cultures were incubated at $28 \pm 0.5^{\circ} \mathrm{C}$ for 14 days. After this period, conidia were harvested and counted using a Neubauer chamber in an optical microscopy at $40 \mathrm{X}$.

\section{Substrate Mixtures}

Mixtures of wheat bran and sugar cane bagasse were prepared in the following proportions $(\mathrm{w} / \mathrm{w})$ : 100/0, 70/30, 50/50, 30/70. Humidity was adjusted to $66 \%$ in vials containing one gram of these mixtures prior to sterilization and inoculation as described above. Conidial production was determined in triplicate after 14 days from the samples for every mixture.

\section{Inoculum level}

Medium was prepared by taking one gram of wheat bran as mentioned above. Three levels of inoculation were assayed: $1 \times 10^{6}, 7 \times 10^{6}$ and $5 \times$ $10^{7}$ conidia/gds. Bottles were incubated at $28{ }^{\circ} \mathrm{C}$ for up to 18 days; every $24 \mathrm{~h}$ conidial yields were determined as described above for three independent bottles from each treatment. Results were adjusted to the best fit to the exponential model described below by the Solver routine (Excel, Microsoft) adjusting the values for $\mathrm{y}_{\max }$ and $k$. Then, the time value (h) for achieving a level of $1 \times 10^{10}$ conidia/gds was calculated; this value was referred to as $\boldsymbol{t}_{\mathbf{1 0}}$. The exponential model was the following:

$$
\frac{d y}{d t}=k \mathrm{e}^{-k \mathrm{t}}\left(\mathrm{y}_{\max }-\mathrm{y}_{0}\right) \text {, resolving for: }
$$

$\mathbf{t}=\mathbf{0}, \mathbf{y}=\mathbf{y}_{\mathbf{0}} ;$ and for $\mathbf{t} \rightarrow \infty, \mathbf{y} \rightarrow \mathbf{y}_{\max }$, the corresponding equation is as follows:

$\mathrm{y}=\mathrm{y}_{0}+\left(\mathrm{y}_{\max }-\mathrm{y}_{0}\right) *\left(1-\mathrm{e}^{-k \mathrm{t}}\right)$

Where:

$\mathbf{y}_{\mathbf{0}}$ : Initial inoculum (conidia/gds).

$\mathbf{y}_{\text {max }}$ : Maximal yield (conidia/gds).

y: Yield at time " $\mathrm{t}$ " (conidia/gds).

t: Time (h).

$\boldsymbol{k}$ : Specific production rate $(1 / \mathrm{h})$.

\section{Hydrophobicity}

This was determined by the phase exclusion method (Boucias et al. 1988; Jeffs and 
Khachatourians, 1997). Fresh harvested conidia suspension was centrifuged at $8000 \mathrm{x}$ g during 30 min; conidial pellet were then washed twice with deionized water at $50^{\circ} \mathrm{C}$ before suspension in acetate buffer $(0.1 \mathrm{M}, \mathrm{pH} 6.0)$. This was mixed with equal volumes of toluene and refrigerated overnight. Then aqueous phase was excluded from the organic phase and was measured at $600 \mathrm{~nm}$ using the same acetate buffer as a blank. Hydrophobicity was calculated using following equation:

$H=\frac{\left(A_{0}-A_{m}\right)}{A_{0}} * 100$

Where:

H: Conidia hydrophobicity (\%)

$\mathbf{A}_{\mathbf{0}}$ : Blank absorbance

$\mathbf{A}_{\mathrm{m}}$ : Aqueous phase absorbance

\section{Viability}

Analysis of viability were carried out in Petri dishes in triplicate with SDA (4\%) medium containing yeast extract $(1 \mathrm{~g} / \mathrm{L})$ and sodium deoxycolate $(1 \mathrm{~g} / \mathrm{L})$ which made the colonies more compact for easy counting. $\mathrm{pH}$ was adjusted to 6.0 using $\mathrm{HCl}(1 \mathrm{~N})$ solution. Petri dishes were inoculated with $200 \mu \mathrm{L}$ of conidial suspension from a proper dilution in order to have 300 conidia per plate. Plates were incubated at $28 \pm 0.5^{\circ} \mathrm{C}$ for 3 days and then number of colonies was counted. The conidia viability was calculated using following equation:

$\mathrm{V}=(\mathrm{n} / 300) * 100$

Where:

V: Conidia viability (\%)

n: Colony number

\section{Statistics Analysis}

Conidia production as well as every measured variable were tested using one way ANOVA analysis by the means of NCSS statistic software with a significant coefficient $\alpha=0.05$.

\section{RESULTS and DISCUSSION}

\section{Effect of water activity}

Wheat bran could support the growth of $B$. bassiana when used as the only nutrient in the medium. Radial extension rate was considered as an estimation of the fungal ability to colonize the substrate on which it was growing. The values for radial extension rate markedly enhanced (between 10 and $15 \%$ ) after the addition of liquid media reported by Alves et al. (2002) or a standard Dextrose Sabouraud broth. This behavior was similar to that reported by Kamp and Bidochka (2002) who found better growth for B. bassiana in more complex media. Our B. bassiana strain was very sensitive to variation in water activity $\left(a_{w}\right)$ since radial extension rate decreased from $77 \mu \mathrm{m} / \mathrm{h}$ (at $\left.\mathrm{a}_{\mathrm{w}}=1.0\right)$ down to $15 \mu \mathrm{m} / \mathrm{h}\left(\right.$ at $\left.\mathrm{a}_{\mathrm{w}}=0.981\right)$ in sole wheat bran medium (Fig. 1), with no detectable growth at $\mathrm{a}_{\mathrm{w}}$ values lower than 0.97 for all the analyzed media. These findings were in accordance to Gervais et al. (1988) who worked with other filamentous fungi such Penicillium roqueforti and Trichoderma viride and described that a value of $\mathrm{a}_{\mathrm{w}}$ close to 1.0 was the best for fungal growth. Nevertheless, for some Aspergillus strains able to grow in solid fermentation, a reduction of $\mathrm{a}_{\mathrm{w}}$ from 0.995 down to 0.965 had a much less marked effect on specific growth rate (Loera and Viniegra-González, 1998; MontielGonzález et al. 2004).

\section{Moisture content}

Solid-state fermentation allows the manipulation of $a_{w}$ values in order to adjust this parameter to particular requirements by fungal strain as mentioned above. The strain of $B$. bassiana showed the better growth at $\mathrm{a}_{\mathrm{w}}=1.0$. In order to analyze the conidial yields at $\mathrm{a}_{\mathrm{w}}=1.0$, moisture content was assayed at three levels in wheat bran medium: $66 \%$ (minimum value to assure $\mathrm{a}_{\mathrm{w}}=1.0$ ), $75 \%$ and $80 \%$. The effect of culture moisture on conidial yields is showed in the Fig. 2. Conidial yield decreased one order of magnitude when moisture content increased from 66 to $80 \%$, with values of $1.18 \pm 0.34\left(\mathrm{x} 10^{10}\right)$ conidia/gds and 1.85 $\pm 0.21\left(\mathrm{x} 10^{9}\right)$ conidia/gds, respectively. This finding could be related to the fact that when water content was raised in the substrate, pores were filled and oxygen mass transfer coefficient diminished (Pandey 2003; Durand et al. 1988). The best relative humidity $(66 \%)$ found in this study was similar to those values reported for conidia production by B. bassiana in other media. These reported media included the mixtures of waste potatoes residues and sugar cane bagasse (60-40\%) (Dalla-Santa et al. 2004; 2005), or wheat bran and rice straw (7:3, w/w) (Kang et al. 2005). 


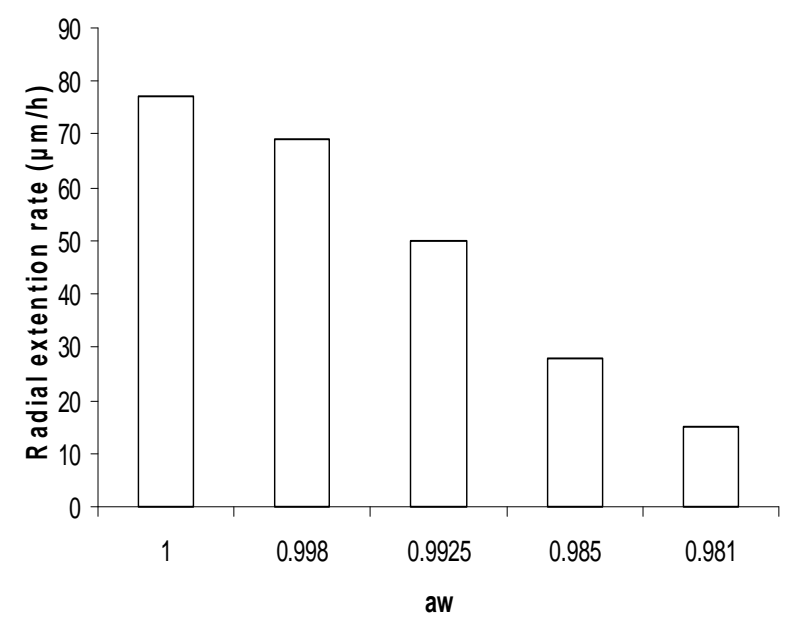

Figure 1- Effect of water activity $\left(\mathrm{a}_{\mathrm{w}}\right)$ on $B$. bassiana radial extention rate $(\mu \mathrm{m} / \mathrm{h})$ grown on wheat bran agar medium with Ethylene glycol as depressor. Results showed are means of triplicates and observed variations were within $12 \%$ of means values.

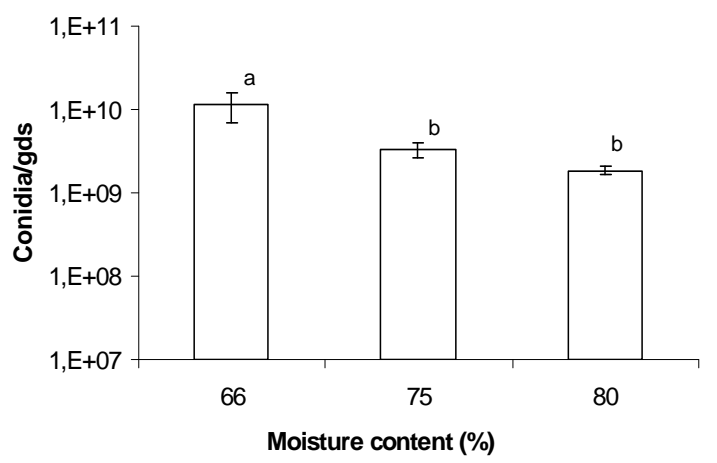

Figure 2 - Effect of moisture content $\left(\mathrm{a}_{\mathrm{w}}=1\right)$ on Beauveria bassiana conidial yields (conidia/gds) in SSF with wheat bran as substratum. Columns with different letters showed significant differences $(P<0.05)$.

\section{Substrate mixtures}

Sugar cane bagasse can function as a texturiser by increasing the porosity and mass transfer during SSF. Table 1 shows the effect of this texturiser in mixtures with wheat bran on conidial yield. Reductions in conidial yields were proportional to the amount of sugar cane bagasse added to every mixture; in fact, in the mixture with the highest amount of sugar cane bagasse $(70 \%)$, the production was $2.6 \pm 0.9\left(\mathrm{x} 10^{9}\right)$ conidia/gds, which represented a reduction of $77 \%$ compared to that with no addition of sugar cane baggase. However, when yield was referred to as conidia per gram of wheat bran, independently to the amount of texturiser, yields did not present significant differences among the mixtures, with average production close to $1 \times 10^{10}$ conidia/gds (Table 1). This behavior was similar to a report by Arzumanov et al. (2005), who described that in SSF using ground rice and sugar cane bagasse (50:50), Metarhizium anisopliae grew almost exclusively on rice particles, and the yield of conidia per gram of total dry matter was lower than that obtained in SSF with only rice as a substrate. 
Table 1 - Effect of substrate mixtures between wheat bran (WB) and sugar cane bagasse (SCB) on conidial yields (conidia/gds). Yields results are also presented in terms of WB alone. Different letters mean significant differences $(P<0.05)$

\begin{tabular}{ccc}
\hline $\begin{array}{c}\text { WB / SCB } \\
(\%)\end{array}$ & $\begin{array}{c}\text { Total yield } \\
(\mathrm{x} \mathrm{10})\end{array}$ & $\begin{array}{c}\text { Yield on WB } \\
(\mathrm{x} \mathrm{10})\end{array}$ \\
\hline $100 / 0$ & $11.5 \pm 0.44 \mathrm{a}$ & $11.5 \pm 0.44 \mathrm{a}$ \\
$70 / 30$ & $6.65 \pm 0.49 \mathrm{~b}$ & $9.50 \pm 0.70 \mathrm{a}$ \\
$50 / 50$ & $4.90 \pm 1.84 \mathrm{~b}$ & $9.80 \pm 3.68 \mathrm{a}$ \\
$30 / 70$ & $2.60 \pm 0.99 \mathrm{~b}$ & $8.33 \pm 3.30 \mathrm{a}$ \\
\hline
\end{tabular}

Inoculum levels: It is convenient for SSF systems to know the effect of inoculum size on the final values for any relevant parameter. Fig. 3 showed that the maximal conidia levels were $1.08 \pm 0.19$ $\left(\mathrm{x} 10^{10}\right), 1.20 \pm 0.82\left(\mathrm{x} 10^{10}\right)$ and $1.53 \pm 1.10(\mathrm{x}$ $10^{10}$ ) conidia/gds for inoculum sizes of $1 \times 10^{6}, 7 \mathrm{x}$ $10^{6}$ and $5 \times 10^{7}$ conidia/gds, respectively. After adjusting the data to the exponential model, there was a reduction in time at which a level of $1 \times 10^{10}$ conidia/gds was achieved, referred to as $\boldsymbol{t}_{10}$, as a function of inoculum levels with the specific production rate values $(k)$ as shown in Table 2 .

For instance, $\boldsymbol{t}_{10}$ was $285 \mathrm{~h}$ using the lowest inoculum ( 1 x $10^{6}$ conidia/gds); however, this value could be reduced to $20 \%$ and $50 \%$ for higher inocula, since values for $\boldsymbol{t}_{10}$ were 232 and $148 \mathrm{~h}$ using $7 \times 10^{6}$ or $5 \times 10^{7}$ conidia/gds, respectively. A level of $10^{7}$ conidia/gds is commonly recommended as inoculum in the cultures of entomophogenic fungi (Dalla-Santa et al. 2004; 2005; Arzumanov et al. 2005). This result suggested that $\boldsymbol{t}_{10}$ could be an appropriate criterion to define the harvest time for an entire batch, which in turn might reduce the maintenance and costs in the processes related to conidia production by entomophogenic fungi.

Table 2 -Values for $k$ according to different inoculum levels.

\begin{tabular}{lc}
\hline Inoculum level (Conidia/gds) & $\boldsymbol{k} \mathbf{x} \mathbf{1 0}^{-3}(\mathbf{1} / \mathbf{h})$ \\
\hline $1 \times 10^{6}$ & 3.85 \\
$7 \times 10^{6}$ & 4.83 \\
$5 \times 10^{7}$ & 3.94 \\
\hline
\end{tabular}

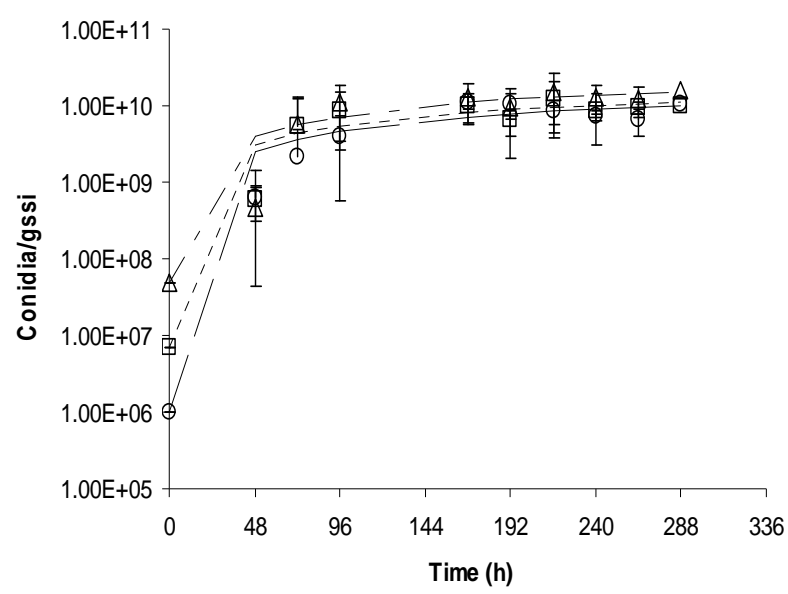

Figure 3 - Inoculum size (conidia/gds) effect on conidia production by Beauveria bassiana in SSF with wheat bran as substratum: $\circ, 1 \times 10^{6} ; \square, 7 \times 10^{6} ; \Delta, 5 \times 10^{7}$. Solid lines: data best fit to model. 


\section{Hydrophobicity and viability}

In order to ensure the compatibility of biocontrol agents with formulation and application techniques, it is necessary to produce the lipophilic (hydrophobic) conidia which suspend easily in oils (Arzumanov et al. 2005). Also, the attachment of conidia of the entomopathogenic fungi to host insects represents the initial event in the establishment of mycosis; this conidium-cuticle interaction may involve a complex of specific (e.g., glycoprotein) and nonspecific (e.g., electrostatic or hydrophobic) recognition mechanisms (Boucias et al. 1988). Thus, hydrophobicity, as well as viability, is related to the quality of conidia for entomopathogenic fungi (Alavo and Accodji 2004; Talaei-Hassanloui et al. 2006). These parameters were not significantly affected as a function of inocula size when determined at 7 days of culture. The values for hydrophobicity were within 90 and $92 \%$, whereas viability averages were around $70 \%$ for all the cultures

\section{CONCLUSION}

It could be concluded that $B$. bassiana was a very sensitive fungus in terms of $a_{w}$ variations, and the highest values of extension growth rate were obtained at $\mathrm{a}_{\mathrm{w}}=1.0$, with no detected growth at $\mathrm{a}_{\mathrm{w}}$ lower than 0.97 . In SSF, $a_{w}=1.0$ could be reached with a moisture content of $66 \%$ and any increase in moisture affected negatively the conidia production using wheat bran. The use of sugar cane bagasse as a texturiser in SSF was not suitable for the production of conidia. Inoculum size could reduce the time at which the maximal conidial productions were achieved with no effect on variables related to conidia quality. This finding could be relevant for prediction of harvesting times using a parameter such as $\boldsymbol{t}_{10}$ described in this study.

\section{ACKNOWLEDGEMENTS}

This work was financially supported by the Mexican Science and Technology Council (CONACyT, SEP-2003-C02-42931) and the Universidad Autónoma Metropolitana. O. NuñezGaona held a scholarship from CONACyT (Registration number 7751).

\section{REFERENCES}

Alavo TBC, Accodji M. (2004). The entomopathogenic fungus Verticillium lecanii (Deuteromycetes, Moniliaceae). The proteins hydrophobins and the biological control of Aphids (Homoptera, Aphididae): literature review. Arch Phytopathol Plant Protect., 37, 201-204.

Alves SB, Rossi LS, Lopes RB, Tamai MA, Pereira R.M. (2002). Beauveria bassiana yeast phase on agar medium and its pathogenicity against Diatraea saccharalis (Lepidoptera Crambidae) and Tetranychus urticae (Acari: Tetranychidae). J Invertebr Pathol., 81, 70-77.

Arcas JA; Díaz BM; Lecuona RE. (1999). Bioinsecticidal activity of conidia and dry mycelium preparations of two insolates of Beauveria bassiana against the sugarcane borer Diatraea saccharalis. $J$ Biotech., 67, 151-158.

Arzumanov T, Jenkins N, Roussos S. (2005). Effect of aeration and substrate moisture content on sporulation of Metarhizium anisopliae Var. acridum. Process Biochem., 40, 1037-1042.

Barranco-Florido JE, Alatorre-Rosas R; GutiérrezRojas M; Viniegra-González G; Saucedo-Castañeda, G. (2002). Criteria for the selection of strains of entomopathogenic fungi Verticillium lecanii for solid state cultivation. Enzyme Microbial Technol., 30, 910915.

Boucias, D; Pendland JC, Latge JP. (1988). Nonspecific factors involved in attachment of entomopathogenic deuteromycetes to host insect cuticle. Appl. Envirom. Microbiol., 54, 1795-1805.

Dalla-Santa HS, Dalla-Santa OR, Brand D, Vandenberghe LPS, Soccol CR. (2005). Spore Production of Beauveria bassiana From AgroIndustrial Residues. Braz Arch Biol Techn., 48, 51-60

Dalla-Santa HS, Sousa NJ, Brand D, Dalla-Santa OR, Pandey A, Sobotka M, Paca J, Soccol CR. (2004). Conidia production of Beauveria sp. by solid state fermentation for biocontrol of Ilex paraguariensis caterpillars. Folia Microbiol., 49, 418-422.

Durand A, Pichon P, Desgranges C. (1988). Approaches to KLa measurements in solid state fermentation. Biotechnol Tech., 2, 11-16.

Feng KC, Liu L Tzeng MY. (2000). Verticilliun lecanii spore production in solid-state and liquid-state fermentations. Bioprocess Eng., 23, 25-29.

Fuguest R, Vey A. (2004). Comparative analysis of the production of insecticidal and melanizing macromolecules by strains of Beauveria spp.: in vivo studies. J Invertebr Pathol 85: 152-167.

Fuxa JR. (1987). Ecological considerations for the use of entomopathogens in IPM. Annu Rev Entomol., 32, 2225-251. 
Gervais P, Molin P, Grajek W, Bensoussan M. (1988). Influence of the water activity of a solid substrate on the growth rate and sporogenesis of filamentous fungi. Biotechnol Bioeng., 31, 457-463.

Jeffs LB, Khachatourians GG. (1997). Estimation of spore hydrophobicity for members of the genera Beauveria, Metarhizium, and Tolypocladium by saltmediated aggregation and sedimentation. Canadian J. Microbiol., 43, 23-28.

Kamp AM, Bidochka MJ. (2002). Conidia production by insect pathogenic fungi on commercially available agars. Lett Appl Microbiol., 35, 74-77.

Kang SW, Lee SH, Yoon CS, Kim SW. (2005). Conidia production by Beauveria bassiana (for the biocontrol of a diamondback moth) during solid-state fermentation in a packed-bed bioreactor. Biotechnol Lett., 27, 135-139.

Larroche C. (1996). Microbial growth and sporulation behaviour in solid state fermentation. J Sci Ind Res., 55, 408-423.
Loera O, Viniegra-González G. (1998). Identification of growth phenotypes in Aspergillus niger pectinase overproducing mutants using image analysis procedures. Biotechnol Tech., 12, 801-804.

Montiel-González AM, Viniegra-González G, Fernández FJ, Loera O. (2004). Effect of water activity on invertase production by improved diploids strains of Aspergillus niger. Process Biochem., 39, 2085-2090.

Pandey A. (2003). Solid-state fermentation. Biochem Eng J., 13, 81-84.

Talaei-Hassanloui R, Kharazi-Pakdel A, Goettel M, Mozaffari J. (2006). Variation in virulence of Beauveria bassiana isolates and its relatedness to some morphological characteristics. Biocontrol Sci Technol., 16, 525-534.
Received: April 14, 2008; Revised: October 03, 2008; Accepted: October 21, 2009. 